\title{
Signatures of Polar-cap Current Flow in Polarization Angle Sweeps
}

\author{
Johann A. Hibschman \\ Jonathan Arons \\ University of California, Berkeley. 601 Campbell Hall, Berkeley \\ $94720-3411$.
}

\begin{abstract}
Upon re-examining the characteristic polarization angle sweep of rotation-powered pulsars, we find that the sweep is perceptibly shifted by aberrational effects and by polar-cap current flow. In addition to the previously known phase shift of the entire sweep by $\Delta \Phi=-4 r / R_{L}$, aberration shifts the polarization angle itself by $\Delta \Psi=$ $-(10 / 3)\left(r / R_{L}\right) \cos \alpha$. Similarly, current flow above the polar cap shifts the polarization sweep by $\Delta \Psi=(10 / 3)\left(r / R_{L}\right)\left(J / J_{G J}\right) \cos \alpha$, potentially providing a method of directly measuring the magnitude of the current. The competition between these two effects produces a potentially observable signature in the polarization angle sweep.
\end{abstract}

\section{Introduction}

Most pulsars emit radiation containing a significant linearly polarized component. Over the course of a pulse, the observed polarization angle of this component changes, forming the characteristic Radhakrishnan and Cooke S-curve (Radhakrishnan \& Cooke 1969).

The theoretical understanding of this curve depends on three assumptions about the pulsar emission mechanism. First, radiation is beamed along the magnetic field. Second, the polarization of the emission is along (or at a fixed angle to) the radius of curvature of the magnetic field. Third, the magnetic field is a dipole. In this work, we relax the first and third assumptions in an attempt to bring the phenomenological model closer to a real physical model.

The effects we consider tend to scale as $r / R_{L}$, where $r$ is the height above the polar cap and $R_{L}$ is the light cylinder radius, the radius at which an object rotating along with the pulsar would be moving at the speed of light. Since the amplitude is inversely proportional to $R_{L}$, we expect these effects to be strongest for millisecond pulsars (MSPs), since they have the smallest values of $R_{L}$.

\section{Perturbations}

We considered two main perturbations: aberrational effects and the effects of polar-cap current flow. Both effects perturb the paths of the emitting particles, 

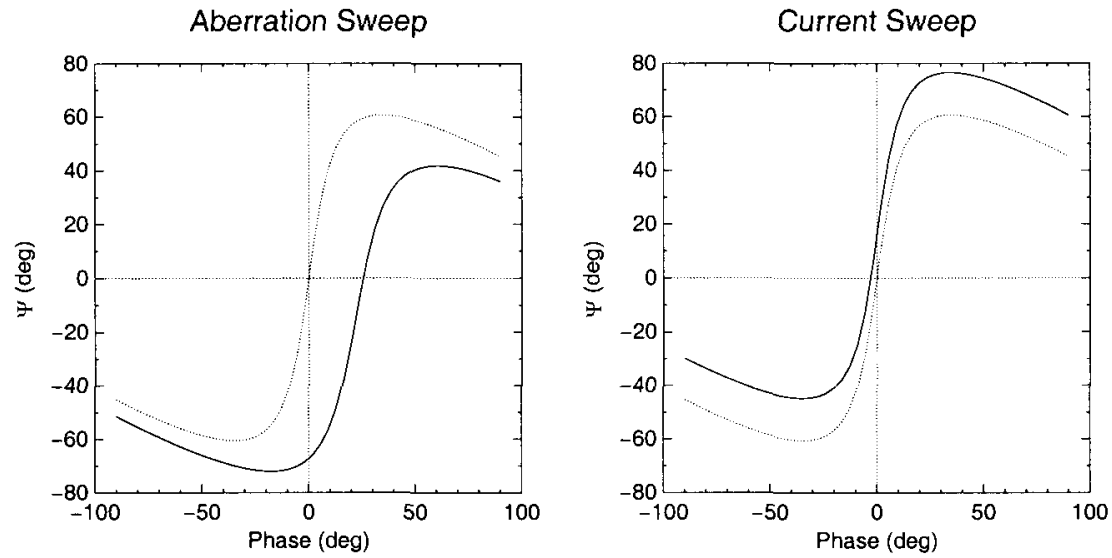

Figure 1. Polarization shifts due to aberration and current-flow. The dotted line represents the unperturbed polarization angle sweep, while the solid is the perturbed. The assumed geometry is $\alpha=30^{\circ}, \beta=5^{\circ}$, $r / R_{L}=0.1$.

which changes the direction of the radius of curvature, changing the expected polarization angle.

\subsection{Aberration}

Aberrational effects relax the assumption that emission is beamed along the magnetic field. The emission of relativistic particles is beamed along their velocities, not the magnetic field. Since the particles co-rotate with the star, they have components of velocity both along the direction of rotation and along the field lines. This additional rotational velocity changes the observed polarization angle.

This aberration produces two primary effects. First the polarization sweep is shifted in phase (with respect to the center of the emission) by

$$
\Delta \Phi=4 \frac{r}{R_{L}},
$$

as independently found by Blaskiewicz, Cordes, \& Wasserman (1991). In addition, in a plot of polarization angle vs. phase, the polarization sweep is shifted downwards by

$$
\Delta \psi=-\frac{10}{3} \frac{r}{R_{L}} \cos \alpha,
$$

where $\alpha$ is the angle between the rotation axis and the magnetic dipole.

\subsection{Field-aligned Current Flow}

Most models of pulsar electrodynamics predict an electric current flow along the open field lines above the pulsar polar cap. Electrostatic acceleration of charges from the neutron star surface generates a "typical" current equal to the Goldreich-Julian charge density moving at the speed of light. 


\section{Return Current}
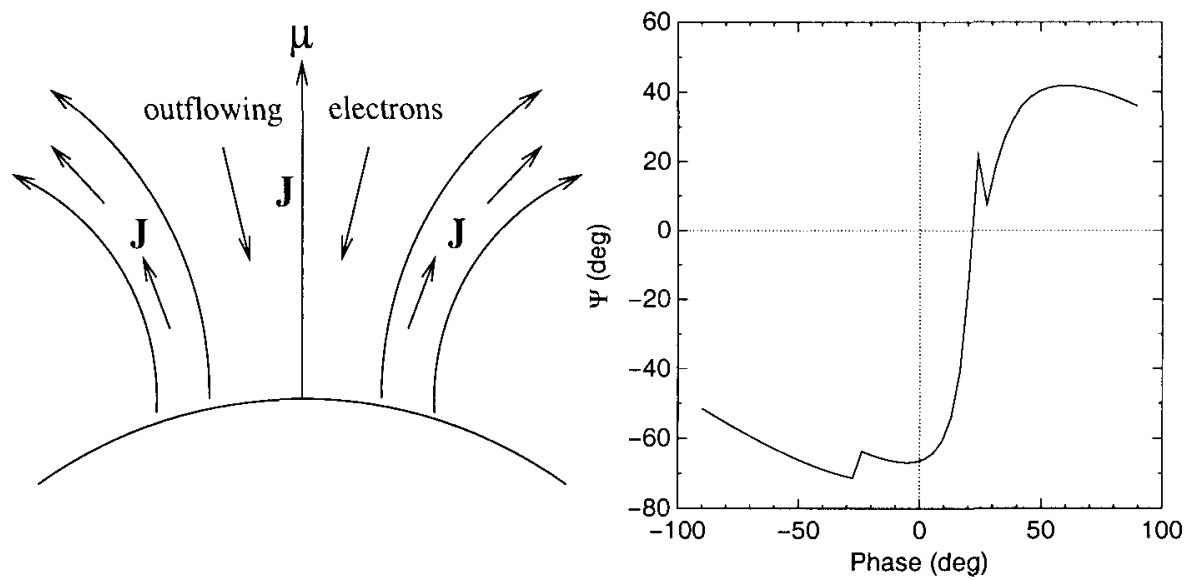

Figure 2. The assumed geometry of the return-current layers and the resulting polarization shift.

This current induces a toroidal perturbing magnetic field, which shifts the observed polarization angle upwards (in a plot of polarization angle vs. phase) by

$$
\Delta \psi=\frac{10}{3} \frac{r}{R_{L}} \frac{J}{J_{G J}} \cos \alpha,
$$

where $J_{G J}$ is the Goldreich-Julian fiducial current, $J_{G J}=\Omega \cdot B / 2 \pi$.

If the current above the polar cap equals the Goldreich-Julian fiducial current, the aberrational and current shifts exactly cancel, except for the aberrational phase shift. Any remaining offset would provide a measurement of the difference between the actual current density and $J_{G J}$.

\section{Return Current Layers}

In this section, we examine a specific model of current flow above a polar cap, using the results of the previous sections. The ring of last closed field lines may carry a thin layer of current, equal and opposite to the total current flow within the ring. In the regions outside this current layer, the auroral current would cancel the perturbative effects of any interior current flow, leaving only the aberrational effects.

This produces a sharp jump in polarization angle as the line of sight passes through the auroral return current layer. Inside the layer, the aberrational and the current effects offset each other, while outside the layer, only the aberrational effect remains. Since the return current layer is assumed to be thin, these jumps are sharp. A broad region of return current flow would produce a much more gradual transition.

Although the return current layer is not normally thought of as a site of emission, it may be visible either through direct emission, by some form of 
two-stream instability, or through refraction or scattering of radiation emitted within the cone. Sharp transitions like the ones described above would be strong evidence for emission in this region or radiation transfer through it.

\section{Conclusion}

The essential conclusion of this work is that detailed polarization studies can reveal information about the structure of the emitting regions of pulsars. The polarization is affected both by current flow over the polar cap and by aberrational effects

If the radius of emission is linked to the frequency of emission, then the polarization sweeps at different frequencies should differ both in phase, due to aberration, and in absolute offset, due to both aberration and current. This absolute difference gives a direct measurement of the magnitude of the current flow above the polar cap.

In the specific model of an illuminated auroral return current layer, singlefrequency measurements would show abrupt transitions in polarization angle as the observer's line of sight passes through the current layer. In other current geometries, we would still expect transitions to exist, with magnitudes on the order of $\left(r / R_{L}\right)\left(J / J_{G J}\right)$.

\section{References}

Blaskiewicz, M., Cordes, J.M., \& Wasserman, I. 1991, ApJ, 370, 643

Radhakrishnan, V., \& Cooke, D.J. 1969, Astrophys. Lett., 3, 225 